\title{
MLS POINT CLOUD SEGMENTATION BASED ON FEATURE POINTS OF SCANLINES
}

\author{
Ryohei Honma ${ }^{1}$, Hiroaki Date ${ }^{2}$, Satoshi Kanai ${ }^{2}$ \\ ${ }^{1}$ Asia Air Survey Co.,Ltd., 215-0004 Kawasaki-shi, Kanagawa, Japan - ryh.honma@ajiko.co.jp \\ ${ }^{2}$ Graduate School of Information Science and Technology, Hokkaido University, 060-0814 Sapporo, Japan - \\ (hdate, kanai)@ssi.ist.hokudai.ac.jp
}

Commission II, WG II/3

KEY WORDS: Point Cloud, MLS, Scanline, Segmentation, Road

\begin{abstract}
:
Point clouds acquired using Mobile Laser Scanning (MLS) are applied to extract road information such as curb stones, road markings, and road side objects. In this paper, we present a scanline-based MLS point cloud segmentation method for various road and road side objects. First, end points of the scanline, jump edge points, and corner points are extracted as feature points. The feature points are then interpolated to accurately extract irregular parts consisting of irregularly distributed points such as vegetation. Next, using a point reduction method, additional feature points on a smooth surface are extracted for segmentation at the edges of the curb cut. Finally, points between the feature points are extracted as flat segments on the scanline, and continuing feature points are extracted as irregular segments on the scanline. Furthermore, these segments on the scanline are integrated as flat or irregular regions. In the extraction of the feature points, neighboring points based on the spatial distance are used to avoid being influenced by the difference in the point density. Based on experiments, the effectiveness of the proposed method was indicated based on an application to an MLS point cloud.
\end{abstract}

\section{INTRODUCTION}

The demand for maps for autonomous driving has recently increased. Therefore, point clouds acquired using Mobile Laser Scanning (MLS) are applied to extract road information such as curb stones, road markings, and road side objects (Zhang et al., 2016). The segmentation of MLS point clouds is required for the extraction of road information. Because the accuracy of the segmentation affects the results of the final extraction of the road information, segmentation with high accuracy is required.

Many segmentation methods for point clouds have been proposed, and are based on unorganized points or scanlines. As a segmentation method of unorganized point clouds, supervoxel based method (e.g. Zai et al., 2018, Kang et al., 2018), region growing based method (e.g. Vieira et al, 2005, Vo et al., 2017), model fitting based method (e.g. Schnabel et al., 2007) etc. were proposed, they are used for extraction of 3D road boundaries from MLS point cloud, extraction of various objects in the urban area from aircraft LiDAR data and TLS point cloud, etc. The main advantage of an unorganized point-based method is applicability any point clouds. In contrast, there are certain disadvantages, for example, the computational cost is high and it is difficult to apply such methods to point clouds of a complex road environment, in addition, the difference in point density and scanline interval owing to the characteristics of the MLS and running speed reduce the segmentation accuracy.

The fundamental ideas of the scanline-based segmentation were published by (Jiang et al., 1994) and (Jiang et al., 1999). Their algorithm first divides each row of a range image into straight line segments, and then applies a region growing process using these line segments. Many applications have extended these methods. Gonzálvez et al. (Gonzálvez et al., 2007) developed an approach for converting a laser scanner point cloud into a realistic $3 \mathrm{D}$ polygonal model, aiming at the automatic $3 \mathrm{D}$ modeling of the basic structures of the medieval wall. Hebel et al. (Hebel et al., 2008) proposed a scanline-based method of airborne laser scanner data, aiming at a fast pre-classification and segmentation of buildings in urban environments. Miyazaki et al. (Miyazaki et al., 2017) proposed a scanline-based region growing method for extracting flat regions such as roads, curbstones and sidewalks from MLS point clouds. Ishikawa et al. (Ishikawa et al., 2018) adopted a scanline-based method toward the extraction and classification of curb types from lowdensity points.

Scanline-based methods for MLS point clouds have advantages in that it is easy to extract the edges of the objects and realize efficient data processing; in addition, they are not influenced by the driving speed during the measurement. However, the interval between points (point density) on the scanlines captured by MLS drastically changes depending on the distance from the scanner and the orientation of the object surfaces. To achieve robust segmentation for various road side objects, a point density insensitive algorithm is required. Nevertheless, the existing methods do not discuss the point density. Furthermore, in related studies, the application of an MLS point cloud segmentation method based on a scanline is limited to road and curb extraction, and there are no methods for dividing point clouds into various objects in a complex environment.

In this paper, we present a scanline-based MLS point cloud segmentation method for various road and road side objects for extracting road information. Our method is based on the feature point extraction of scanlines considering the point density, and targets various objects in a complex environment. The scanlines are divided into flat and irregular segments. Furthermore, flat and irregular regions are generated according to the integration of the neighboring segments.

The remainder of this paper is organized as follow. In section 2, the proposed method is explained in detail. Section 3 shows the experimental results of applying the proposed method to real MLS point clouds. The final section provides some concluding remarks and areas of future study. 


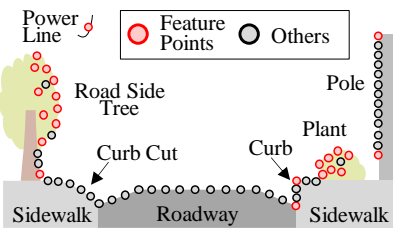

(a) Feature Point Extraction

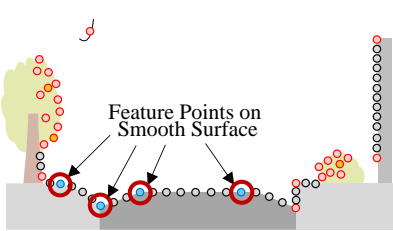

(c) Feature Point Extraction on Smooth Surfaces

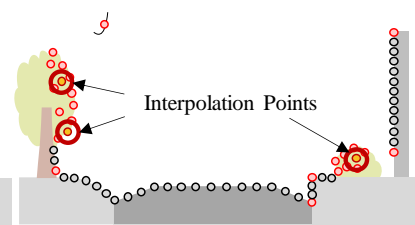

(b) Feature Point Interpolation
Figure 1. Method of MLS Point Cloud Segmentation

\section{PROPOSED METHOD}

\subsection{Outlines}

In the point cloud used in our study, each point has an acquisition time, and a sequence of points ordered by the acquisition time is used as the scanline. The laser scanner uses the time-of-flight technology, and multi-echo points with the same acquisition time are included in the scanline. The scanline on the road is tilted by approximately $45^{\circ}$ from the operating direction of the vehicle.

One of the final applications of MLS point cloud segmentation is to recognize road and road side objects, such as sidewalks, poles, traffic signs, plants, and road side trees. In this paper, a basic segmentation method for MLS point clouds, which can extract flat regions such as road surfaces and complex regions such as plants, is proposed. For this purpose, the point clouds are segmented into flat and irregular regions such as plants. The proposed method consists of feature point extraction when considering the difference in the point density, a division of scanlines into segments, and an integration of segments to extract each region. The outline of the method is shown in Fig. 1. First, boundary points of objects, called "feature points," are extracted, as shown in Fig. 1 (a). The feature points are then interpolated to accurately extract irregular parts consisting of irregularly distributed points such as vegetation, as shown in Fig. 1 (b). Next, using a point reduction method, additional feature points on a smooth surface such as edges of the curb cut are extracted, as shown in Fig. 1 (c). Finally, irregular regions such as vegetation and flat regions such as the grounds and walls of buildings are extracted through an integration of the neighbor scanlines with similar features, as shown in Fig. 1 (d).

\subsection{Feature Point Extraction}

The final goal in this study is to generate regions for each road object. Therefore, the points used as the boundary of objects with a smooth surface on the scanline are extracted as the feature points. In this step, 1) end points of the scanline, 2) jump edge points, and 3) corner points are extracted as feature points.

\section{- End Points of the Scanline}

The end points of the scanline, which are the boundary points of unmeasured parts caused by no return pulses such as the sky, e.g., traffic signs or edges of the walls, are extracted based on

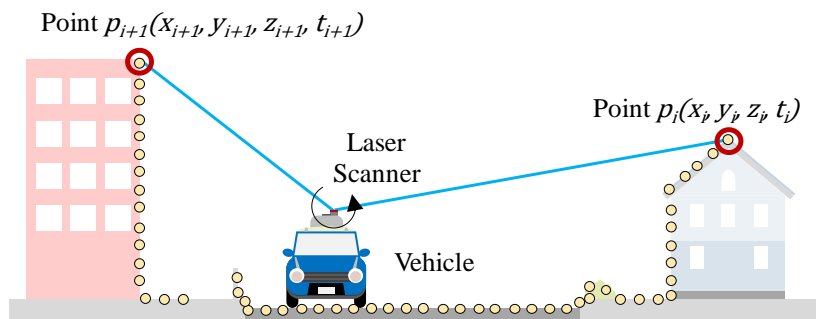

Figure 2. End Points of Scanline

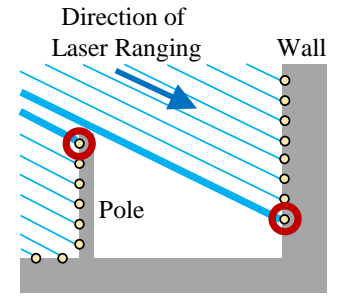

(a) Boundary Points on Scanline

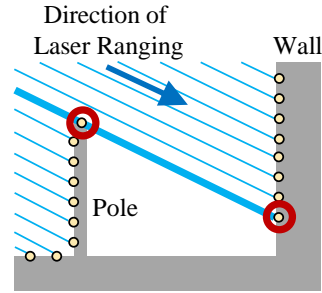

(b) Points of Same Acquisition Time
Figure 3. Jump Edge Points

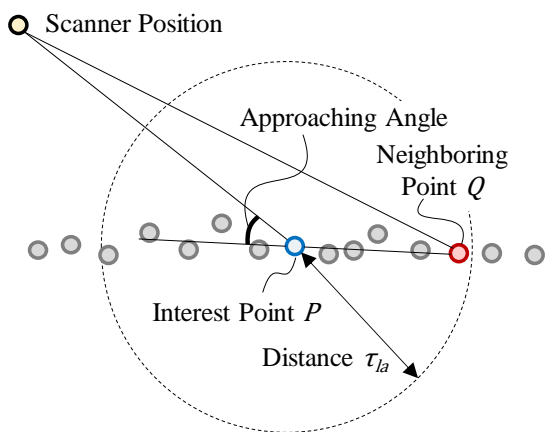

Figure 4. Approaching Angle

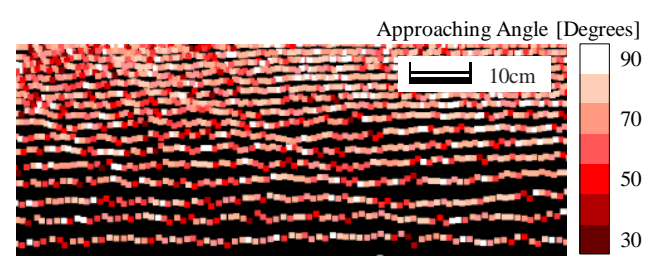

(a) Calculation Results of Using Nearest Points

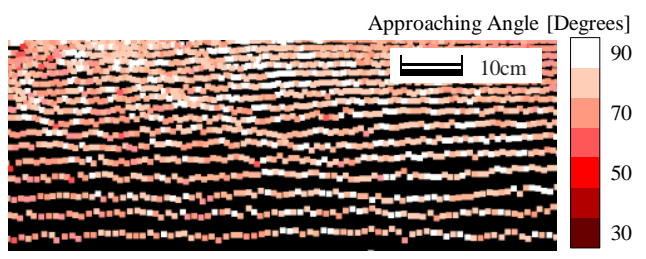

(b) Calculation Results of Using Farthest Points

Figure 5. Influence of Approaching Angle Calculation Using Neighbouring Point Selection Method

thresholding for differences in the acquisition times of the neighboring points on the scanline (see Fig. 2).

\section{- Jump Edge Points}

The point at which the distance between points is drastically increased is also the dividing point of the objects. This point is defined as a jump edge point. For example, when the points of a pole and a wall of a building behind the pole exist on a scanline, 


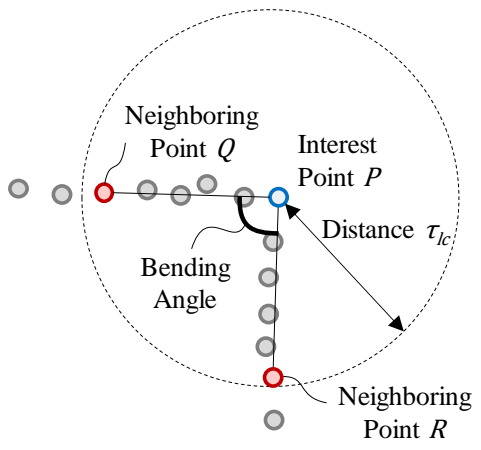

Figure 6. Bending Angle

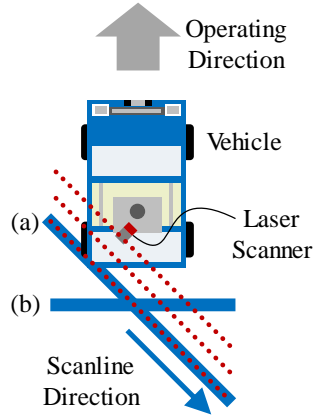

(a) View of Cross Section in the Scanline Direction

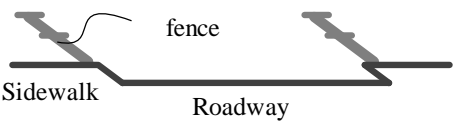

(b) View of Cross Section at the Vertical to the Operating Direction of Vehicle

Figure 7. Point Cloud Projection at the Operating Direction of Vehicle

the boundary points of the pole and the wall of the building are defined as the jump edge points (see Fig. 3 (a)). However, because the distance between points on the scanlines captured by MLS changes depending on the distance from the scanner and the orientation of the object surfaces, the approaching angle is used to detect the jump edge points. In addition, points with the same acquisition time are detected as jump edge points (see Fig. 3 (b)).

In this study, we define the approaching angle, as shown in Fig. 4. Points with an approaching angle of less than threshold $\tau_{a}$ are detected as jump edge points. The approaching angles calculated using neighboring points on the scanline are sensitive to scanning noise at the region close to the scanner where the point intervals are extremely small. As shown in Fig. 4, to prevent the false detection of jump edge points caused by the noise sensitivity of the approaching angles, the farthest point within the fixed distance $\tau_{l a}$ from point $P$ is set as a neighbouring point $Q$ in the approaching angle calculation. The approaching angle calculated using the closest point at the high point density locations, such as below the scanner, may not be properly valued, as shown in Fig. 5 (a). However, it is possible to calculate the approaching angle without the influence of the point density and ranging errors by using the farthest point within the fixed distance, as shown in Fig. 5 (b).

\section{- Corner Points}

The intersection part of two planes, such as a roadway and curb, or the sidewalk and wall of a building, is the boundary of the object. Because the intersections appear as corners on the scanline, a point with a large bending angle is defined as a corner point. The bending angle of the surface at point of interest $P$ is calculated using point $P$ and its neighboring points $Q$ and $R$, as shown in Fig. 6. Points with a bending angle of less than threshold $\tau_{c}$ are detected as corner points. To realize a stable calculation of the bending angles against the point density differences, similar to the approaching angle calculation, the

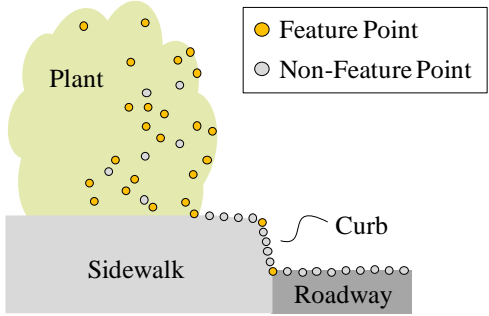

Figure 8. Distribution of Feature and Non-Feature Points in Irregular Parts

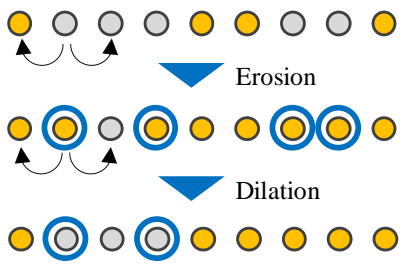

Figure 9. Closing in Morphology

neighboring points $Q$ and $R$ are selected as the farthest points within the distance threshold $\tau_{l c}$ from point $P$.

As shown in Fig. 7, extraction of the corner points is applied on a scanline projected onto a plane perpendicular to the running direction of the vehicle to detect the corner points of curbs without an influence of the mounted angle of the scanner.

\subsection{Interpolation of Feature Points}

The bending angle of the points in the irregular parts, such as a vegetation area, can be large. There are also a number of jump edge points based on the same acquisition time in the irregular parts. The feature points are then dominantly distributed within the irregular parts, as shown in Fig. 8. To improve the segmentation accuracy of irregular parts, the closing of the morphology, which consists of erosion and dilation operations, is performed. As shown in Fig. 9, non-feature points in which either or both neighbor points are feature points are converted into feature points. Next, feature points in which either or both neighbor points are non-feature points are converted into feature points. As a result, non-feature points in the section where feature points are dominantly distributed are converted into feature points.

\subsection{Feature Point Extraction on Smooth Surface}

To finally segment the roadway and sidewalk, it is necessary to divide the point cloud on a smooth surface such as edges of a curb cut at an intersection or crosswalk (see Fig. 10). Therefore, points between the feature points are further segmented using additional feature points obtained by the Douglas-Peucker algorithm (Douglas and Peucker, 1973). This algorithm divides the point sequence recursively according to the distance from the straight-line segments approximating the point sequence. In our method, a sequence of successive non-feature points and its two boundary feature points is divided using the algorithm. With the algorithm, a straight line connecting the two feature points, which are the boundary of the point sequence, is then generated. The point $P_{\max }$ with the largest distance $d_{\max }$ from the line connecting the feature points is extracted, as shown in Fig. 11(a). If the distance $d_{\max }$ is equal to or greater than the threshold value $\tau_{d}$, the line segment is divided by the point $P_{\max }$, as shown in Fig. 11(b). Furthermore, division lines are divided recursively until there is no division point by the above 


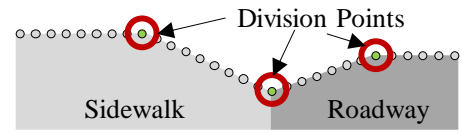

Figure 10. The Division Points on Smooth Surface

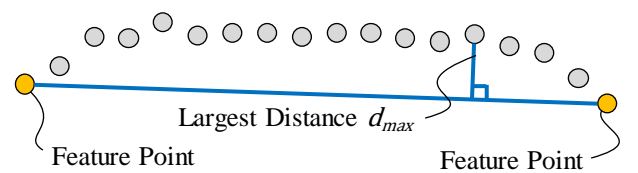

(a) Extraction of the Point with the Largest Distance

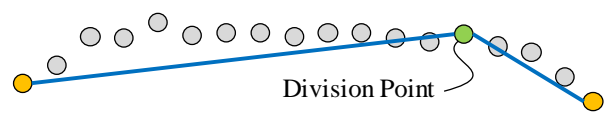

(b) Division of the Line Segment

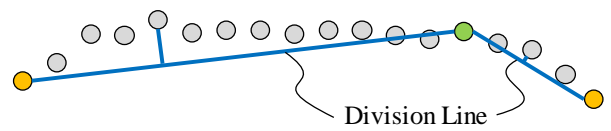

(c) Extraction of the Division Points on the Division Lines

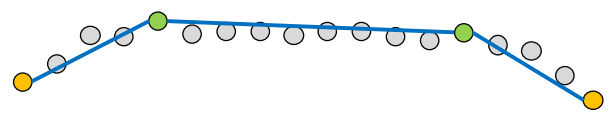

(d) Final Result

Figure 11. Feature Points Extraction on Smooth Surface

processing, as shown in Fig. 11(c) (d). Extracted division points of the straight-lines are defined as the feature points.

\subsection{Extraction of Irregular and Flat Regions}

During this process, irregular and flat segments, which are the point sequences on the scanlines, are first defined using the feature points, and the irregular and flat regions are then extracted by integrating the segments.

A sequence of non-feature points and its boundary between two feature points are identified as a segment. The segments with fewer points than threshold $\tau_{i}$ are detected as irregular segments, and other segments are detected as flat segments, as shown in Fig. 12(a). The slope angle of the flat segments is then calculated. Next, the flat segments on neighboring scanlines, which are close to each other, and whose difference in slope angles is less than threshold $\tau_{m}$, are integrated as the flat regions, as shown in Fig. 12(b). In addition, non-integrated segments during this process are classified as irregular segments.

As a result of the above process, the segments with a few points and the non-integrated segments remain as irregular segments. Next, irregular segments that are closed to each other are integrated as irregular regions, as shown in Fig. 12(c).

\section{EXPERIMENTS}

The point cloud used in the experiments was collected using an MLS named StreerMapper. Although two RIEGL VQ-250 laser scanners are mounted on StreetMapper, only the point cloud

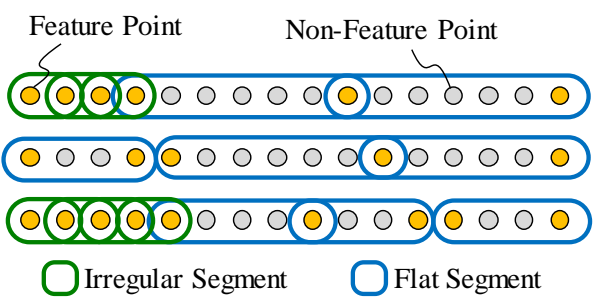

(a) Detection of Irregular and Flat Segments

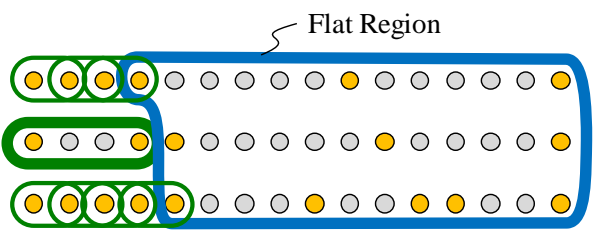

(b) Integration of Flat Segments

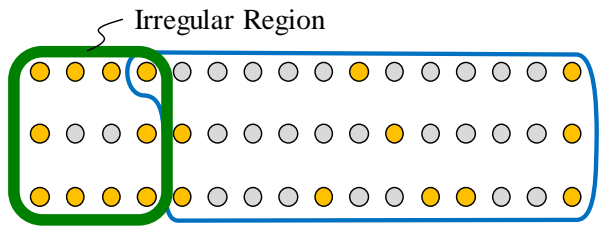

(c) Integration of Irregular Segments

Figure 12. Division of Irregular Region and Flat Region

acquired from one laser scanner is used in these experiments. The laser scanner makes use of the time-of-flight distance measurement principle. In this experiment, the pulse rate of each laser scanner was $200 \mathrm{kHz}$, and the scanning rate was 100 rps. The laser scanner was mounted approximately $2 \mathrm{~m}$ above the ground on the vehicle. The scan line interval was 30 to 60 $\mathrm{mm}$, and the interval of the points was approximately $10 \mathrm{~mm}$ at the ground just below the scanner and approximately $40 \mathrm{~mm}$ at the ground $5 \mathrm{~m}$ away from the scanner.

The scan sites are in Sapporo city, Japan. One of the two scan sites is a road with three lanes, as shown in Fig. 13(a). There are sidewalks, poles, road side trees, and plants on the left side of the road. There are median strips, highway bridge piers, and girders on the right side of the road. There are also traffic lights, signs, and power lines above the road. At this site, the vehicle was driven in the central lane. Another scan site is a street with two lanes, as shown in Fig. 13(b). There are sidewalks and houses on both sides of the roadway, and poles and plants present on the sidewalks. At this site, the point cloud is sparse on the right side of the figure because the point cloud is acquired using the MMS vehicle from the left lane.

The experimental parameters are summarized in Table 1. The threshold for the differences in the acquisition times used to extract the end points was set as sufficiently smaller than the pulse rate. The threshold of the approaching angle used to extract the jump edge points was set as a very small value. The threshold of the bending angle to extract the corner points was set as a value of less than $90^{\circ}$. The minimum distance for the calculation of the approaching angle and the surface angle was set with reference to the size of the road object. Other parameters were set experimentally. 


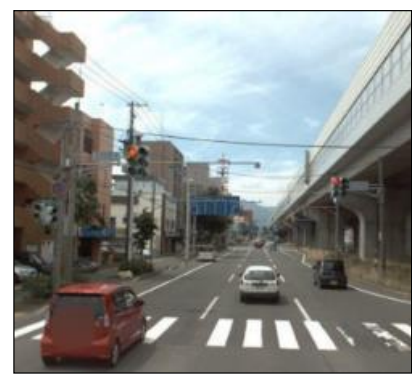

(a) Site A

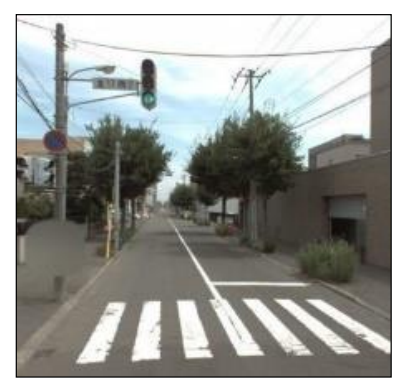

(b) Site B
Figure 13. Scan Sites Used in this Experiment

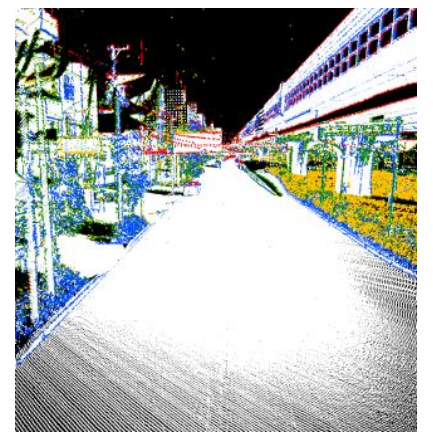

(a) Site A

End Points of Scanline Jump Edge Points by Calculation of Approach Angle Jump Edge Points by Same Acquisition Time Corner Points

Figure 14. Extraction Results of Feature Points

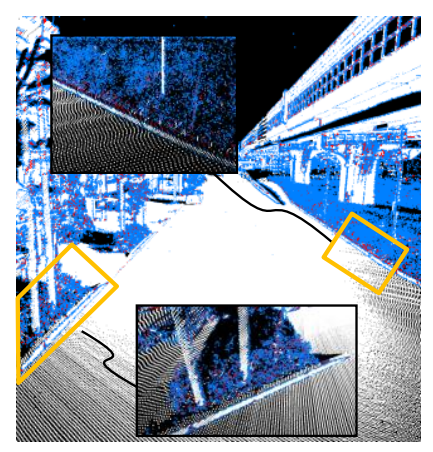

(a) Site A

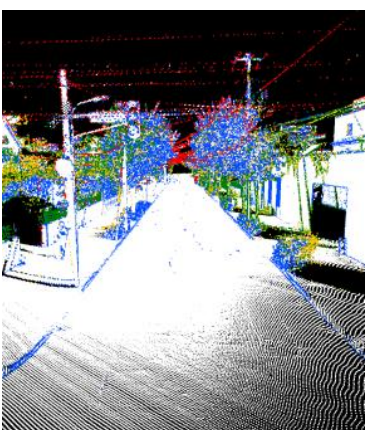

(b) Site B
Table 1. Parameters Used in this Experiment

\begin{tabular}{|c|c|c|}
\hline Parameter & Symbol & Value Unit \\
\hline Threshold for End Points Extraction & $\tau_{t}$ & 10 points \\
\hline Threshold of Approach Angle & $\tau_{a}$ & 3 degrees \\
\hline Distance for Approach Angle Calculation & $\tau_{l a}$ & $80 \mathrm{~mm}$ \\
\hline Threshold of Bend Angle & $\tau_{c}$ & 150 degrees \\
\hline Distance for Bend Angle Calculation & $\tau_{l c}$ & $80 \mathrm{~mm}$ \\
\hline Kernel Size at the Closing Process & $K$ & 3 points \\
\hline Threshold for Douglas-Peucker Algorithm & $\tau_{d}$ & $20 \mathrm{~mm}$ \\
\hline Threshold for Irregular Segments Extraction & $\tau_{i}$ & 2 points \\
\hline Threshold for Flat Segments Integration & $\tau_{m}$ & 5 degrees \\
\hline
\end{tabular}

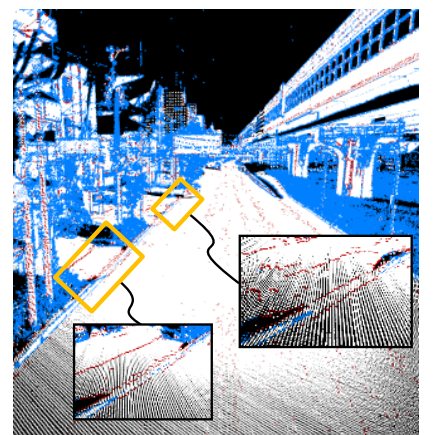

(a) Site A

Feature Points Division Points

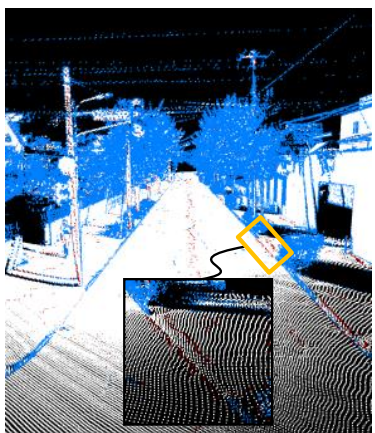

(b) Site B
Figure 16. Extraction Results of Division Points on Smooth Surface

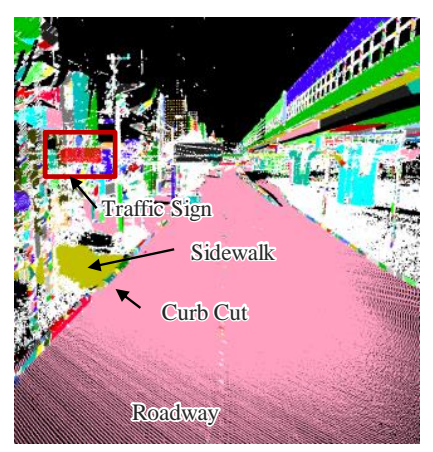

(a) Site A

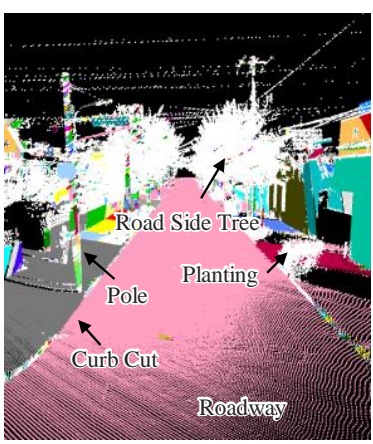

(b) Site B
Figure 15. Interpolation Results between Feature Points

Fig. 14 shows the results of the feature point extraction. In this figure, the end points of the scanline, the jump edge points acquired by judgement of the multi-echo points, the jump edge points by evaluation of approaching angle, the corner points, and other points are colored in red, orange, green, blue, and white, respectively. Most of the end points of the scanline are the points of a wire or edge of a building. Most of the jump edge points of the multi-echo points are located in areas with vegetation. Multi-echo points exist at the boundary of an object too. Most of the jump edge points based on a calculation of the approaching angle are the located boundary of the poles and the objects behind them. Most of the corner points are located in parts with vegetation and curb edges. There are no feature points on a curb cut.

Fig. 15 shows the results of the feature point interpolation. In this figure, the feature points, interpolated points, and other points are colored in blue, red, and white, respectively. Many non-feature points remaining between the feature points of plants or street trees were interpolated. However, slight non-
Figure 17. Extraction Results of Irregular and Flat Regions

feature points were observed in the parts with plants or street trees after this process.

Fig. 16 shows the results of the division point extraction on a smooth surface. In this figure, the feature points and interpolated points, the additional feature points on a smooth surface, and other points are colored in blue, brown, and white, respectively. The points where the slope angle changes slightly a smooth surface. The division points were also extracted near the boundary of curb cut. However, approximately 30\% of division positions of the curb cut were not accurate and had a shift to approximately $50 \mathrm{~mm}$ in the across direction.

Fig. 17 shows the extraction results of irregular and flat regions. In the figure, the points of the irregular regions are colored in white, and the points of the flat regions are colored using random colors other than white. The road surface was extracted as a single flat region. The curbs, sidewalks, walls of the buildings, traffic signs, and bridge piers were composed as flat regions. at the road and sidewalk were extracted as the division points on 
Table 2. Segmentation Accuracy at Site A

\begin{tabular}{ccccc}
\hline Region & $\begin{array}{c}\text { Total Number } \\
\text { of Regions }\end{array}$ & Success Rate & $\begin{array}{c}\text { Rate of Over } \\
\text { Segmentation }\end{array}$ & $\begin{array}{c}\text { Rate of Under } \\
\text { Segmentation }\end{array}$ \\
\hline Flat Region & 285 & $77.2 \%(220)$ & $14.4 \%(41)$ & $9.1 \%(26)$ \\
Irregular Region & 27 & $33.3 \%(9)$ & $11.1 \%(3)$ & $66.7 \%(18)$ \\
\hline
\end{tabular}

Table 3. Segmentation Accuracy at Site B

\begin{tabular}{ccccc}
\hline Region & $\begin{array}{c}\text { Total Number } \\
\text { of Regions }\end{array}$ & Success Rate & $\begin{array}{c}\text { Rate of Over } \\
\text { Segmentation }\end{array}$ & $\begin{array}{c}\text { Rate of Under } \\
\text { Segmentation }\end{array}$ \\
\hline Flat Region & 210 & $67.1 \%(141)$ & $9.5 \%(20)$ & $24.3 \%(51)$ \\
Irregular Region & 41 & $34.1 \%(14)$ & $7.3 \%(3)$ & $58.5 \%(24)$ \\
\hline
\end{tabular}

The accuracy of the segmentation was evaluated using the ground truth, which was manually produced. During the experiments, over segmented regions, and under segmented regions were evaluated. An over-segmented region is defined as a region consisting of multiple extracted regions with a sufficient number of points. If an extracted region includes sufficient numbers of points of multiple manually segmented regions, the regions are identified as under-segmented regions. The over or under-segmentation rate is defined as the number of over or under-segmented regions divided by the number of total regions. In the evaluation of flat regions, roadways, sidewalks, walls of buildings, and flat parts of bridges were used. As irregular regions, plants and tree crown were evaluated.

Tables 2 and 3 show the quantitative evaluation results of the extraction accuracy at sites A and B. In the tables, the numbers in parentheses represent the number of corresponding regions. The number of correctly-segmented regions in the success rate is the total number of regions that are neither under nor oversegmentation. Some regions are evaluated as both the under and over segmented regions. The main factor of the over segmentation for flat regions is that there are small regions in the region of the roadway and the sidewalk. The main factor of the under segmentation for flat regions is the influence of some regions of the sidewalk with a curb cut being integrated into the region of the roadway. The rate of under segmentation is high because the sparse points on road surfaces and walls behind plants and tree crowns were often included in the extracted irregular segments (see Fig. 18).

The processing time for approximately 12 million points in a road of approximately $800 \mathrm{~m}$ in length was 24.1 seconds on a PC with a $4.2 \mathrm{GHz}$ CPU Core i7.

\section{CONCLUSIONS}

In this study, we proposed an MLS point cloud segmentation method based on the feature points of the scanlines. In this method, the scanlines are first segmented by using feature points. The initial feature points are extracted by thresholding for three criteria, and they are modified by using morphologybased interpolation and a point reduction method. Then the regions are generated according to the integration of the neighboring segments. The proposed method realizes an MLS point cloud segmentation of irregular and flat regions. We applied this method to point clouds acquired by a MMS in two sites. In our experiments, the average success rates of flat and irregular region extraction were $72.9 \%$ and $33.8 \%$, respectively. Because feature points on a smooth surface are extracted during the segmentation process of the scanlines, roadway and sidewalk parts bounded by curb cuts are well divided as respective flat regions. On the other hand, under-segmentation for many irregular regions $(62.8 \%)$ was observed caused by the sparse points behind objects. Future studies will include an

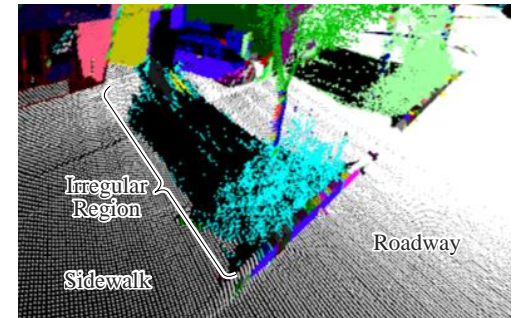

Figure 18. Example of Under Segmentation for Irregular Regions. The irregular region that colored in cyan includes sparse points on the sidewalk.

improvement of the segmentation accuracy and an extension for applying our method to various objects such as pole like objects.

\section{REFERENCES}

Douglas D., Peucker T., 1973, Algorithms for the reduction of the number of points required to represent a digitized line or its caricature, The Canadian Cartographer, 10(2), pp. 112-122.

Gonzálvez P.R., Aguilera D.G., Lahoz J.G., 2007, From point cloud to surface: modeling structures in laser scanner point clouds, ISPRS Workshop on Laser Scanning 2007, pp. 338-343.

Hebel M., Stilla U., 2008, Pre-classification of points and segmentation of urban objects by scan line analysis of airborne lidar data, The International Archives of the Photogrammetry, Remote Sensing and Spatial Information Sciences, Vol. 37, pp. 105-110.

Ishikawa K., Kubo D., Amano Y., 2018, Curb detection and accessibility evaluation from low-density mobile mapping point cloud data, International Journal of Automation Technology, 12(3), pp. 376-385.

Jiang X.Y., Bunke H., 1994, Fast segmentation of range images into planar regions by scan line grouping, Machine Vision and Applications, 7(2), pp. 115-122.

Jiang X., Bunke H., 1999, Edge detection in range images based on scan line approximation, Computer Vision and Image Understanding, 73(2), pp. 183-199.

Kang Z., Yang J., 2018, A probabilistic graphical model for the classification of mobile LiDAR point clouds, ISPRS Journal of Photogrammetry and Remote Sensing, Vol.143, pp. 108-123.

Miyazaki R., Yamamoto M., Harada K., 2017, Line-based planar structure extraction from a point cloud with an anisotropic distribution, International Journal of Automation Technology, 11(4), pp. 657-665.

Schnabel R., Wahl R., Klein R., 2007, Efficient RANS AC for point-cloud shape detection, Computer Graphics Forum, 26(2), pp. 214-226.

Vieira M., Shimada K., 2005, Surface mesh segmentation and smooth surface extraction through region growing, Computer Aided Geometric Design, 22(8), pp. 771-792.

Vo A.V., Truong-Hong L., Laefer D., Bertolotto M., 2015 , Octree-based region growing for point cloud segmentation, ISPRS Journal of Photogrammetry and Remote Sensing, Vol. 104, pp. 88-100. 
Zai D., Li J., Guo Y., Cheng M., Lin Y., Luo H., Wang C., 2018, 3-D road boundary extraction from mobile laser scanning data via supervoxels and graph cuts, IEEE Transactions on Intelligent Transportation Systems, 19(3), pp. 802-813.

Zhang H., Li J., Cheng M., Wang C., 2016, Rapid inspection of pavement markings using mobile LiDAR point clouds, The International Archives of the Photogrammetry, Remote Sensing and Spatial Information Sciences, Vol. XLI-B1, pp. 717-723. 